\title{
Kinematik und Dynamik räumlicher Stöße von Kugeln
}

Die dreidimensionale Dynamik allgemeiner, zusammenstoßender Körper ist kompliziert und erlaubt im Zusammenhang mit der jeweiligen Kontaktmechanik des Stoßes in der Regel weder eine analytische Behandlung noch eine auch nur ansatzweise überschaubare Darstellung der vollständigen Lösung. Im Rahmen dieses Buches soll der allgemeine räumliche Stoß deswegen nur für den vergleichsweise einfachen Fall von Kugeln behandelt werden ${ }^{1}$. Das vorliegende Kapitel ist daher der makroskopischen Dynamik des räumlichen ZusammenstoBes zweier Kugeln gewidmet. Es wird dabei besonders Wert darauf gelegt, die bei den - für eine analytische Behandlung notwendigen - Vereinfachungen der Bewegungsgleichungen getroffenen mechanischen Annahmen in ihrem Sinn und dem Grad der Beschränkung, die sie der Betrachtung auferlegen, klar darzulegen. Ähnliche Herleitungen und Darstellungen finden sich beispielsweise bei Brach [1], Jäger [2], Brilliantov et al. [3], Stronge [4] und Bar-Lev [5].

\subsection{Bewegungsgleichungen}

\subsubsection{Geometrie und Notation}

Eine schematische Skizze des Zusammenstoßes zweier Kugeln ist in Abb.2.1 gezeigt. Die Kugeln haben die Radien $R_{1}$ und $R_{2}$, die Massen $m_{1}$ und $m_{2}$ und die Trägheitsmomente bezüglich des eigenen Schwerpunkts $J_{1}^{S}$ und $J_{2}^{S}$. Die Körper müssen nicht unbedingt

\footnotetext{
${ }^{1}$ Für den reinen zentrischen Normalstoß (die makroskopische Dynamik ist in diesem Fall trivial), werden hingegen allgemeine axialsymmetrische Körper, z.B. auch Kegel oder zylindrische Flachstempel, behandelt. 
Abb. 2.1 Schema des räumlichen Stoßes zweier Kugeln. Doppelpfeile bezeichnen Rotationsvektoren. Die Kugeln sind nur als Schnittansicht in der Stoßebene dargestellt

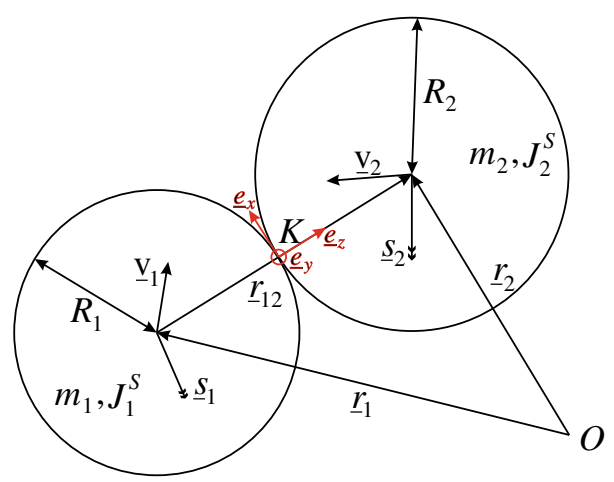

homogen sein, sollen aber zumindest eine kugelsymmetrische Dichteverteilung aufwei$\mathrm{sen}^{2}$. Die Positionen, Geschwindigkeiten und Spins der Kugeln seien in einem raumfesten Koordinatensystem durch die Vektoren $\underline{r}_{i}, \underline{v}_{i}$ und $\underline{s}_{i}:=R_{i} \underline{\omega}_{i}$, mit den Vektoren der Winkelgeschwindigkeit $\underline{\omega}_{i}$, gegeben. An dieser Stelle und für den weiteren Teil dieses Kapitels bezieht sich der Index $i$ auf die beiden Kugeln und kann entsprechend die Werte „1“ und „2“ annehmen.

Der Verbindungsvektor zwischen den beiden Kugelschwerpunkten,

$$
\underline{r}_{12}:=\underline{r}_{2}-\underline{r}_{1}
$$

definiert den Normalenvektor der momentanen Kontaktfläche während des Stoßes, $\underline{e}_{z}$, als

$$
\underline{e}_{z}:=\frac{\underline{r}_{12}}{r_{12}}
$$

wobei der Abstand zwischen den beiden Schwerpunkten durch

$$
r_{12}=R_{1}+R_{2}-d
$$

bestimmt ist. Hier bezeichnet $d$ die Indentierungstiefe während des Stoßes. Der Normalenvektor rotiert während der Kollision mit einer Winkelgeschwindigkeit $\underline{\Omega}$ und hat daher die Zeitableitung

$$
\underline{\dot{e}}_{z}=\underline{\Omega} \times \underline{e}_{z}
$$

Es soll angenommen werden, dass das deformierte Gebiet gegenüber den Abmaßen der Kugeln als makroskopische Körper klein ist. Dies gewährleistet einerseits, dass die Kugeln ihre makroskopische Form behalten - was die Struktur der Bewegungsgleichungen deutlich

\footnotetext{
${ }^{2}$ Die Trägheitstensoren der Kugeln können in diesem Fall als skalare Vielfache des Einheitstensors geschrieben werden, ihre Komponenten sind also bei einer beliebigen Rotation des Koordinatensystems invariant.
} 
vereinfacht - andererseits sind dann die Gradienten der undeformierten Oberflächen im Kontaktgebiet klein. Dies ist als sogenannte „Halbraumhypothese“ unabdingbare Voraussetzung der kontaktmechanischen Rechnungen im nächsten Kapitel. Es sei $2 a$ die charakteristische Länge des Kontaktgebiets (bei einem kreisförmigen Kontaktgebiet ist $a$ der Radius). Da dies gleichzeitig die charakteristische Tiefe des deformierten Gebiets darstellt ${ }^{3}$, kann die genannte Annahme als

$$
\frac{2 a}{R} \ll 1,
$$

mit einem charakteristischen Radius $R$ von der Größenordnung der beiden Kugelradien, geschrieben werden. Andererseits ist dann im Rahmen dieser Näherung

$$
\frac{d}{R} \approx \frac{a^{2}}{R^{2}} \ll 1 .
$$

Die Differenz der Geschwindigkeiten im Kontaktpunkt $K$,

$$
\underline{v}_{K}:=\underline{v}_{2}-\underline{v}_{1}-\left(\underline{s}_{1}+\underline{s}_{2}\right) \times \underline{e}_{z}
$$

definiert gemeinsam mit $\underline{e}_{z}$ die Stoßebene. Die (rotierende) orthonormale Basis wird daher durch die Einheitsvektoren

$$
\begin{aligned}
& \underline{e}_{y}:=\frac{\underline{e}_{z} \times \underline{v}_{K}}{\left|\underline{e}_{z} \times \underline{v}_{K}\right|}, \\
& \underline{e}_{x}:=\underline{e}_{y} \times \underline{e}_{z}
\end{aligned}
$$

vervollständigt.

\subsubsection{Kinematik und Dynamik}

Die Zeitableitungen der Vektoren $\underline{r}_{12}$ und $\underline{v}_{K}$ sind wegen der Definitionen (2.1), (2.2), (2.4) und (2.7) durch

$$
\begin{aligned}
& \underline{\dot{r}}_{12}=\dot{r}_{12} \underline{e}_{z}+r_{12} \underline{\Omega} \times \underline{e}_{z}, \\
& \ddot{\underline{r}}_{12}=\ddot{r}_{12} \underline{e}_{z}+2 \dot{r}_{12} \underline{\Omega} \times \underline{e}_{z}+r_{12}\left(\underline{\dot{\Omega}} \times \underline{e}_{z}+\underline{\Omega} \times \underline{\Omega} \times \underline{e}_{z}\right), \\
& \underline{\dot{v}}_{K}=\ddot{\underline{r}}_{12}-\left[\underline{\dot{s}}_{1}+\underline{\dot{s}}_{2}+\left(\underline{s}_{1}+\underline{s}_{2}\right) \times \underline{\Omega}\right] \times \underline{e}_{z}
\end{aligned}
$$

\footnotetext{
${ }^{3}$ Die Feldgleichungen der Elastizitätstheorie enthalten keine intrinsischen Längenskalen; die einzige charakteristische Länge des Kontaktproblems ist daher die Länge des Kontaktgebiets.
} 
gegeben. Der Vektor $\underline{\Omega}$ hat keine torsionale Komponente. Nimmt man, wie oben beschrieben, an, dass die Kugelradien zeitlich konstant und sehr viel größer als die Indentierungstiefe sind, kann Gl.(2.11) zu

$$
\ddot{\underline{r}}_{12}=-\left[\ddot{d}+\left(R_{1}+R_{2}\right) \Omega^{2}\right] \underline{e}_{z}-\left[2 \dot{d} \underline{\Omega}-\left(R_{1}+R_{2}\right) \underline{\dot{\Omega}}\right] \times \underline{e}_{z}
$$

vereinfacht werden. Die Impulsbilanz für jede Kugel ist durch

$$
m_{i} \ddot{\underline{r}}_{i}=\underline{F}_{i}
$$

gegeben. Wenn man alle äußeren Einflüsse (z.B. durch die Erdanziehung) gegenüber der Wechselwirkung im Kontakt vernachlässigt, fordert das dritte Newtonsche Axiom dabei, dass

$$
\underline{F}_{1}=-\underline{F}_{2}:=\underline{F} .
$$

Daher bleibt der Impuls des Gesamtsystems aus beiden Kugeln erhalten und die translatorische Bewegung wird durch die Gleichung

$$
\underline{\ddot{r}}_{12}=-\frac{1}{\tilde{m}} \underline{F}
$$

mit der effektiven Masse

$$
\tilde{m}:=\frac{m_{1} m_{2}}{m_{1}+m_{2}},
$$

beschrieben. In der Bilanz der Drehimpulse für beide Kugeln,

$$
J_{i}^{S} \underline{\dot{\omega}}_{i}=(-1)^{i-1} R_{i} \underline{e}_{z} \times \underline{F}_{i}+\underline{M}_{i},
$$

findet das Wechselwirkungsprinzip auch für die Kontaktmomente Anwendung,

$$
\underline{M}_{1}=-\underline{M}_{2}:=\underline{M},
$$

und der Drehimpuls des Gesamtsystems aus beiden Kugeln,

$$
\underline{L}:=\sum_{i=1}^{2}\left(m_{i} \underline{r}_{i} \times \underline{v}_{i}+J_{i}^{S} \underline{\omega}_{i}\right),
$$

ist natürlich ebenfalls eine Erhaltungsgröße, denn

$$
\underline{\dot{L}}=\underline{r}_{1} \times \underline{F}+\underline{r}_{12} \times \underline{F}-\underline{r}_{2} \times \underline{F}=\underline{0} .
$$




\subsection{Vereinfachungen der Bewegungsgleichungen}

\subsubsection{Weitere vereinfachende Annahmen}

Es sei angenommen, dass die Spins und die Geschwindigkeitskomponenten der Kugeln senkrecht zur Normalenachse der Kontaktfläche von der Größenordnung einer charakteristischen Geschwindigkeit $v_{x}$ sind, die nicht deutlich größer als die charakteristische Geschwindigkeit $v_{z}$ in Normalenrichtung ist. Der Stoßwinkel sollte also nicht extrem flach sein ${ }^{4}$. In diesem Fall erhält man für die Rotation der Stoßachse während der Kollision mit der Dauer $T_{S}$ und der maximalen Eindringtiefe $d_{\max }$ die Abschätzung

$$
\Omega T_{S} \approx \frac{v_{x}}{R} \frac{d_{\max }}{v_{z}} \ll 1
$$

Daraus folgt, dass in den Bewegungsgleichungen langsame dynamische Effekte durch zentrifugale oder Coriolis-Terme vernachlässigt werden können. Die kinematischen Gl. (2.13) und (2.12) vereinfachen sich in diesem Fall zu

$$
\begin{aligned}
& \ddot{\underline{r}}_{12}=-\ddot{d} \underline{e}_{z}+\left(R_{1}+R_{2}\right) \underline{\dot{\Omega}} \times \underline{e}_{z}, \\
& \underline{\dot{v}}_{K}=\underline{\ddot{r}}_{12}-\left(\underline{\dot{s}}_{1}+\underline{\dot{s}}_{2}\right) \times \underline{e}_{z} .
\end{aligned}
$$

Die Kontaktmomente sind von der Größenordnung

$$
M \approx a F \ll R F
$$

und damit vernachlässigbar gegenüber den Momenten der Kontaktkräfte bezüglich der Schwerpunkte der Kugeln. Diese Vernachlässigung der Kontaktmomente hat auf den ersten Blick einen Haken: Da die Kontaktkräfte bezüglich der Kugelschwerpunkte kein Moment um die Normalenachse aufbringen, bleibt die Rotation um diese Achse - bei der Vernachlässigung der Kontaktmomente - während des Stoßes erhalten. Dies scheint der Tatsache zu widersprechen, dass eine solche Rotation bei Reibung im Kontakt zu elastischen Torsionsspannungen und einem resultierenden Bohrmoment um die Normalenachse führt. Im Unterkapitel 7.2 wird aber gezeigt werden, dass dieses Moment tatsächlich klein ist, wenn die Kontaktradien klein gegenüber den Kugelradien sind.

Die Vernachlässigung langsamer dynamischer Effekte und der Kontaktmomente führt zu zwei weiteren Erhaltungsgrößen, nämlich der Drehimpulse der beiden Kugeln bezüglich des (im Rahmen der getroffenen Annahmen raumfesten) Kontaktpunktes, denn in diesem Fall ist

$$
\frac{\mathrm{d}}{\mathrm{d} t}\left(J_{i}^{S} \underline{\omega}_{i}+(-1)^{i} m_{i} R_{i} \underline{e}_{z} \times \underline{v}_{i}\right)=J_{i}^{S} \underline{\dot{\omega}}_{i}+(-1)^{i} R_{i} \underline{e}_{z} \times \underline{F}_{i}=\underline{0} .
$$

\footnotetext{
${ }^{4}$ Die Änderungen, die sich für sehr flache Winkel ergeben, werden im Unterkapitel 7.1 diskutiert.
} 
Die Bewegungsgleichung kann man dann zu

$$
\underline{\dot{v}}_{K}=-\frac{1}{\tilde{m}} \underline{F}-\underline{e}_{z} \times \underline{F} \times \underline{e}_{z} \sum_{i=1}^{2} \frac{R_{i}^{2}}{J_{i}^{S}}
$$

zusammenfassen. Es ist hilfreich, die effektiven Werte für den Krümmungsradius,

$$
\frac{1}{\tilde{R}}:=\sum_{i=1}^{2} \frac{1}{R_{i}} \Leftrightarrow \tilde{R}=\frac{R_{1} R_{2}}{R_{1}+R_{2}},
$$

und das Massenträgheitsmoment,

$$
\frac{\tilde{R}^{2}}{\tilde{J}}:=\sum_{i=1}^{2} \frac{R_{i}^{2}}{J_{i}^{S}} \Leftrightarrow \tilde{J}=\frac{J_{1}^{S} J_{2}^{S} \tilde{R}^{2}}{J_{2}^{S} R_{1}^{2}+J_{1}^{S} R_{2}^{2}},
$$

einzuführen. Abschließend sei angenommen, dass die Kontaktkräfte in der von $\underline{e}_{z}$ und $\underline{e}_{x}$ aufgespannten Ebene liegen, weil sich die Beiträge aus den Schubspannungen $\tau_{y z}$ wegen der Symmetrie bezüglich der Stoßebene aufheben. Die Komponenten der Kontaktkräfte seien entsprechend $F_{x}:=\underline{F} \cdot \underline{e}_{x}$ und $F_{z}:=\underline{F} \cdot \underline{e}_{z}$. Aus der Bewegungsgleichung (2.27) folgt in diesem Fall, dass auch $\underline{\dot{v}}_{K}$ in dieser Stoßebene liegt, die entsprechend niemals verlassen wird. Die Bewegungsgleichungen für die drei ebenen Freiheitsgrade,

$$
\begin{aligned}
v_{x} & :=\underline{\dot{r}}_{12} \cdot \underline{e}_{x}, \\
v_{z} & :=\underline{\dot{r}}_{12} \cdot \underline{e}_{z}=-\dot{d}, \\
\omega & :=\frac{1}{\tilde{R}}\left(R_{1} \underline{\omega}_{1}+R_{2} \underline{\omega}_{2}\right) \cdot \underline{e}_{y},
\end{aligned}
$$

lauten dann wegen der Gl. (2.16) und (2.18) wie folgt:

$$
\begin{aligned}
\dot{v}_{x} & =-\frac{F_{x}}{\tilde{m}}, \\
\ddot{d} & =\frac{F_{z}}{\tilde{m}}, \\
\dot{\omega} & =\frac{\tilde{R}}{\tilde{J}} F_{x} .
\end{aligned}
$$

Das dreidimensionale Stoßproblem kann man daher offensichtlich unter den getroffenen Annahmen als ebenen Stoß zwischen einer Kugel mit dem Radius $\tilde{R}$, der Masse $\tilde{m}$ sowie dem Trägheitsmoment $\tilde{J}$ bezüglich des eigenen Schwerpunkts und einem Halbraum betrachten. Dies ist in Abb. 2.2 visualisiert. Dabei wurden die Koordinaten so eingeführt, dass die Komponenten der Kontaktkräfte auf den Halbraum in positive Koordinatenrichtung zeigen. 
Abb. 2.2 Ebener Stoß einer Kugel auf einen Halbraum; schematische Darstellung und Freischnitt

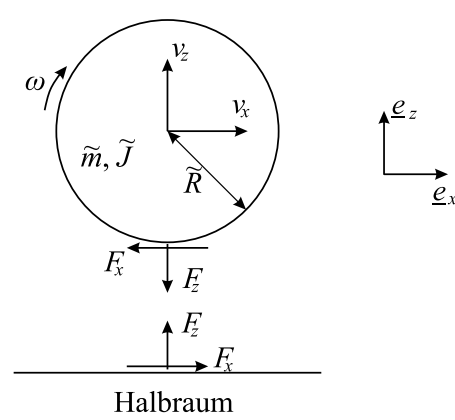

\subsubsection{Die Stoßzahlen}

Es ist klar, dass man im Rahmen der oben getroffenen Vereinfachungen die Stoßzahlen $\epsilon_{z}$ und $\epsilon_{x}$ durch

$$
\begin{array}{ll}
\underline{v}_{K, e} \cdot \underline{e}_{z}=-\epsilon_{z}\left(\underline{v}_{K, 0} \cdot \underline{e}_{z}\right), & \epsilon_{z} \in[0 ; 1], \\
\underline{v}_{K, e} \cdot \underline{e}_{x}=-\epsilon_{x}\left(\underline{v}_{K, 0} \cdot \underline{e}_{x}\right), & \epsilon_{x} \in[-1 ; 1], \\
\underline{v}_{K, e} \cdot \underline{e}_{y}=\underline{v}_{K, 0} \cdot \underline{e}_{y}, &
\end{array}
$$

mit den relativen Geschwindigkeitsvektoren des Kontaktpunktes vor und nach dem Stoß, $\underline{v}_{K, 0}$ und $\underline{v}_{K, e}$, einführen kann. Definiert man die gesamte Änderung der Geschwindigkeit der ersten Kugel während des Stoßes als $\underline{V}$,

$$
\underline{V}:=\underline{v}_{1, e}-\underline{v}_{1,0},
$$

können die Geschwindigkeit der zweiten Kugel und die Spins beider Kugeln nach dem Stoß wegen der Impuls- und Drehimpulserhaltungssätze allein mit diesem Vektor $\underline{V}$ (und den bekannten Vektoren vor dem Stoß) ausgedrückt werden. $\underline{V}$ hat nur eine normale und eine tangentiale Komponente und ist vollständig durch die beiden Stoßzahlen $\epsilon_{z}$ und $\epsilon_{x}$ bestimmt. Die Normalkomponente ist durch

$$
V_{z} \underline{e}_{z}=\frac{\tilde{m}}{m_{1}}\left(1+\epsilon_{z}\right)\left(\underline{v}_{K, 0} \cdot \underline{e}_{z}\right) \underline{e}_{z}
$$

und die tangentiale Komponente durch

$$
V_{x} \underline{e}_{x}=\kappa \frac{\tilde{m}}{m_{1}}\left(1+\epsilon_{x}\right) \underline{e}_{z} \times\left(\underline{v}_{K, 0} \times \underline{e}_{z}\right)
$$

gegeben, wobei das Kürzel 


$$
\kappa:=\frac{j_{1} j_{2}\left(m_{1}+m_{2}\right)}{j_{1} j_{2}\left(m_{1}+m_{2}\right)+j_{1} m_{1}+j_{2} m_{2}}, \quad j_{i}:=\frac{J_{i}^{S}}{m_{i} R_{i}^{2}}
$$

eingeführt wurde. Man kann feststellen, dass der dimensionslose Trägheitsradius, der bei der Behandlung des einzelnen Stoßproblems eine große Rolle spielt, durch die Beziehung

$$
\tilde{j}:=\frac{\tilde{J}}{\tilde{m} \tilde{R}^{2}}=\frac{\kappa}{1-\kappa}
$$

bestimmbar ist. Die Umkehrung dieser Gleichung liefert

$$
\kappa=\frac{\tilde{j}}{1+\tilde{j}} .
$$

Da offensichtlich $\kappa \leq 0,5$ ist, ist damit auch gezeigt, dass $\tilde{J}$ ein physikalisches Trägheitsmoment mit $\tilde{j} \leq 1$ darstellt. Die Zusammenhänge zwischen den Geschwindigkeiten vor und nach dem Stoß sind schließlich durch

$$
\begin{aligned}
& \underline{v}_{1, e}=\underline{v}_{1,0}+\frac{\tilde{m}}{m_{1}}\left[\left(1+\epsilon_{z}\right)\left(\underline{v}_{K, 0} \cdot \underline{e}_{z}\right) \underline{e}_{z}+\kappa\left(1+\epsilon_{x}\right) \underline{e}_{z} \times\left(\underline{v}_{K, 0} \times \underline{e}_{z}\right)\right], \\
& \underline{v}_{2, e}=\underline{v}_{2,0}-\frac{\tilde{m}}{m_{2}}\left[\left(1+\epsilon_{z}\right)\left(\underline{v}_{K, 0} \cdot \underline{e}_{z}\right) \underline{e}_{z}+\kappa\left(1+\epsilon_{x}\right) \underline{e}_{z} \times\left(\underline{v}_{K, 0} \times \underline{e}_{z}\right)\right], \\
& \underline{s}_{1, e}=\underline{s}_{1,0}+\frac{\kappa}{j_{1}} \frac{\tilde{m}}{m_{1}}\left(1+\epsilon_{x}\right)\left(\underline{e}_{z} \times \underline{v}_{K, 0}\right), \\
& \underline{s}_{2, e}=\underline{s}_{2,0}+\frac{\kappa}{j_{2}} \frac{\tilde{m}}{m_{2}}\left(1+\epsilon_{x}\right)\left(\underline{e}_{z} \times \underline{v}_{K, 0}\right)
\end{aligned}
$$

gegeben. Die Umkehrabbildung, also die Bestimmung der Geschwindigkeiten und Spins vor dem Stoß aus den Vektoren nach dem Stoß, ergibt sich aus den oberen Gleichungen durch die Ersetzung der Stoßzahlen mit ihren Inversen. Man erhält die Ausdrücke

$$
\begin{aligned}
& \underline{v}_{1,0}=\underline{v}_{1, e}+\frac{\tilde{m}}{m_{1}}\left[\frac{1+\epsilon_{z}}{\epsilon_{z}}\left(\underline{v}_{K, e} \cdot \underline{e}_{z}\right) \underline{e}_{z}+\kappa \frac{1+\epsilon_{x}}{\epsilon_{x}} \underline{e}_{z} \times\left(\underline{v}_{K, e} \times \underline{e}_{z}\right)\right], \\
& \underline{v}_{2,0}=\underline{v}_{2, e}-\frac{\tilde{m}}{m_{2}}\left[\frac{1+\epsilon_{z}}{\epsilon_{z}}\left(\underline{v}_{K, e} \cdot \underline{e}_{z}\right) \underline{e}_{z}+\kappa \frac{1+\epsilon_{x}}{\epsilon_{x}} \underline{e}_{z} \times\left(\underline{v}_{K, e} \times \underline{e}_{z}\right)\right], \\
& \underline{s}_{1,0}=\underline{s}_{1, e}+\frac{\kappa}{j_{1}} \frac{\tilde{m}}{m_{1}} \frac{1+\epsilon_{x}}{\epsilon_{x}}\left(\underline{e}_{z} \times \underline{v}_{K, e}\right), \\
& \underline{s}_{2,0}=\underline{s}_{2, e}+\frac{\kappa}{j_{2}} \frac{\tilde{m}}{m_{2}} \frac{1+\epsilon_{x}}{\epsilon_{x}}\left(\underline{e}_{z} \times \underline{v}_{K, e}\right) .
\end{aligned}
$$

Für das ebene Modell in Abb. 2.2 lauten die Geschwindigkeiten nach dem Stoß in Abhängigkeit von den beiden Stoßzahlen wie folgt: 


$$
\begin{aligned}
v_{z, e} & =-\epsilon_{z} v_{z, 0} \\
v_{x, e} & =v_{x, 0}-\kappa\left(1+\epsilon_{x}\right)\left(\tilde{R} \omega_{0}+v_{x, 0}\right), \\
\tilde{R} \omega_{e} & =\tilde{R} \omega_{0}-(1-\kappa)\left(1+\epsilon_{x}\right)\left(\tilde{R} \omega_{0}+v_{x, 0}\right) .
\end{aligned}
$$

Während des Stoßes verändert sich die gesamte kinetische Energie um

$$
\begin{aligned}
\Delta U_{\text {kin }} & =\frac{m_{1}}{2}\left(\underline{v}_{1, e}^{2}-\underline{v}_{1,0}^{2}\right)+\frac{m_{2}}{2}\left(\underline{v}_{2, e}^{2}-\underline{v}_{2,0}^{2}\right)+\frac{j_{1} m_{1}}{2}\left(\underline{s}_{1, e}^{2}-\underline{s}_{1,0}^{2}\right)+\frac{j_{2} m_{2}}{2}\left(\underline{s}_{2, e}^{2}-\underline{s}_{2,0}^{2}\right) \\
& =\frac{\tilde{m}}{2}\left[v_{z, K, 0}^{2}\left(\epsilon_{z}^{2}-1\right)+\kappa v_{x, K, 0}^{2}\left(\epsilon_{x}^{2}-1\right)\right] \leq 0
\end{aligned}
$$

Hier bezeichnen $v_{z, K, 0}$ und $v_{x, K, 0}$ die Koordinaten des Vektors der relativen Geschwindigkeit beider Kugeln im Kontaktpunkt vor dem Stoß. Die tangentiale Stoßzahl für die Bewegung des Schwerpunktes (in dem ebenen Modell aus Abb.2.2) lässt sich durch die Beziehung

$$
\epsilon_{x, S}=\frac{\kappa v_{x, K, 0}}{v_{x, 0}}\left(1+\epsilon_{x}\right)-1
$$

mit $\epsilon_{x}$ verknüpfen. Der verallgemeinerte Einfallswinkel $\alpha$ der Bewegung des Kontaktpunktes ist $^{5}$

$$
\tan \alpha=-\frac{v_{x, K, 0}}{v_{z, K, 0}}
$$

Der verallgemeinerte Rückprallwinkel ist entsprechend

$$
\tan \alpha^{*}=\frac{v_{x, K, e}}{v_{z, K, e}}=-\frac{\epsilon_{x}}{\epsilon_{z}} \tan \alpha .
$$

Das Stoßproblem ist damit vollständig gelöst, wenn die beiden Stoßzahlen bekannt sind. Dem Problem der exakten kontaktmechanischen Bestimmung dieser Stoßzahlen unter verschiedenen Umständen sind die folgenden Teile des vorliegenden Buches gewidmet.

\subsection{Zusammenfassung}

Die relative Geschwindigkeit im Kontaktpunkt der beiden Kugeln zum Zeitpunkt der ersten Berührung definiert gemeinsam mit dem Verbindungsvektor der Kugelschwerpunkte die initiale Stoßebene der räumlichen Kollision zweier Kugeln. Unter den folgenden allgemeinen Annahmen kann man die sich aus dem Impuls- und Drehimpulssatz ergebenden

\footnotetext{
${ }^{5}$ Man beachte, dass mit den verwendeten Definitionen $v_{z, K, 0}<0$ sein muss, damit es überhaupt zu einem Zusammenstoß kommt. Ohne Beschränkung der Allgemeinheit sei $v_{x, K, 0}>0$.
} 
Bewegungsgleichungen stark vereinfachen; die ursprüngliche Stoßebene wird unter diesen Annahmen während der Kollision nicht verlassen:

(a) Die Kontaktkräfte sind so groß, dass alle äußeren Einflüsse während des Stoßes vernachlässigt werden können.

(b) Das deformierte Gebiet ist klein gegenüber den Abmaßen der Kugeln als makroskopische Körper. Die Kugeln behalten dann während der Kollision ihre makroskopische Form, die Gradienten der (undeformierten) Oberflächen sind im Kontaktgebiet klein und die Kontaktmomente können gegenüber den Momenten der Kontaktkräfte bezüglich der Kugelschwerpunkte vernachlässigt werden.

(c) Die tangentialen Geschwindigkeitskomponenten und Spins sind nicht von einer höheren Größenordnung als die normalen Geschwindigkeitskomponenten. Unter Berücksichtigung der Annahme (b) folgt daraus, dass man langsame dynamische Effekte aus Zentrifugal- und Coriolis-Beiträgen vernachlässigen kann.

(d) Der Vektor der momentanen Kontaktkraft liegt in der momentanen Stoßebene.

Der Stoß wird unter diesen Annahmen auf eine äquivalente Kollision zwischen einer Kugel mit einer flachen Ebene zurückgeführt. Die Lösung dieses Stoßproblems kann man durch zwei kinematische Stoßzahlen formulieren, die die Verhältnisse der Geschwindigkeiten des Kontaktpunktes nach und vor dem Stoß in normaler, beziehungsweise tangentialer Richtung angeben. Alle Geschwindigkeiten und Spins nach dem Stoß lassen sich für die räumliche Kollision unter den oben genannten Annahmen mithilfe dieser beiden Stoßzahlen ausdrücken.

\section{Literatur}

1. Brach, R. M. (1984). Friction, restitution, and energy loss in planar collisions. Journal of Applied Mechanics, 51(1), 164-170.

2. Jäger, J. (1994). Analytical solutions of contact impact problems. Applied Mechanics Review, 47(2), 35-54.

3. Brilliantov, N. V., Spahn, F., Hertzsch, J. M., \& Pöschel, T. (1996). Model for collisions in granular gases. Physical Review E, 53(5), 5382-5392.

4. Stronge, W. J. (2000). Impact mechanics. Cambridge: Cambridge University Press.

5. Bar-Lev, O. (2005). Kinetic and hydrodynamic theory of granular gases. Dissertation. Tel Aviv University. 
Open Access Dieses Kapitel wird unter der Creative Commons Namensnennung 4.0 International Lizenz (http://creativecommons.org/licenses/by/4.0/deed.de) veröffentlicht, welche die Nutzung, Vervielfältigung, Bearbeitung, Verbreitung und Wiedergabe in jeglichem Medium und Format erlaubt, sofern Sie den/die ursprünglichen Autor(en) und die Quelle ordnungsgemäß nennen, einen Link zur Creative Commons Lizenz beifügen und angeben, ob Änderungen vorgenommen wurden.

Die in diesem Kapitel enthaltenen Bilder und sonstiges Drittmaterial unterliegen ebenfalls der genannten Creative Commons Lizenz, sofern sich aus der Abbildungslegende nichts anderes ergibt. Sofern das betreffende Material nicht unter der genannten Creative Commons Lizenz steht und die betreffende Handlung nicht nach gesetzlichen Vorschriften erlaubt ist, ist für die oben aufgeführten Weiterverwendungen des Materials die Einwilligung des jeweiligen Rechteinhabers einzuholen. 